\title{
Diffuse histoplasmosis in a patient with sarcoidosis
}

\author{
PS Badesha, MG Saklayen, N Hillman
}

\begin{abstract}
Summary
A case is presented of a woman with a long history of sarcoidosis who developed diffuse histoplasmosis. Although association between these two entities has been described before, there are only a few case reports of histoplasmosis among sarcoid patients. Diagnosis was finally established by bone marrow biopsy which saved the patient's life.
\end{abstract}

Keywords: histoplasmosis, sarcoidosis

Histoplasma capsulatum, a dimorphic fungus and a soil saprophyte, is distributed throughout the world. Although histoplasmosis occurs worldwide, endemic regions in the US are in the Ohio-Mississippi River Valley, with microfoci in other areas. ${ }^{1}$ It is usually asymptomatic or self-limiting, except in patients who have a predisposing immunodeficiency.

Disseminated histoplasmosis predominantly affects the lungs and reticuloendothelial system, but it may affect any part of the body. ${ }^{2}$ The diagnosis becomes particularly difficult when systemic diseases such as tuberculosis or sarcoidosis predispose to dissemination of histoplasmosis. ${ }^{3}$ We report a case of diffuse histoplasmosis that developed in a patient with sarcoidosis.

\section{Case report}

A 29-year-old black woman had presented to the hospital with fever and cough of several weeks duration. Pulmonary sarcoidosis was diagnosed by mediastinal lymph node biopsy in 1987, followed by progressive renal failure. A percutaneous renal biopsy revealed focal glomerulosclerosis. The patient's pulmonary symptoms were successfully treated with corticosteroids, but renal function soon deteriorated to end-stage renal-disease requiring haemodialysis. She received a onehaplotype-identical living renal transplant from her mother in 1989. Her graft was functioning well two years post-transplant, when she moved from Boston to Dayton, Ohio. During the second year after her transplant, she inadvertently stopped taking azathioprine for several weeks. Her renal function deteriorated and a renal biopsy revealed severe acute and chronic rejection with prominent eosinophils in the interstitium. No granuloma was visible. Despite pulse solumedrol therapy her graft kidney could not be salvaged and she was started on ambulatory peritoneal dialysis.
Two months later she developed persistent fever with night sweats, chills, diarrhoea and weight loss. There was no evidence of infection or recurrence of sarcoidosis despite an extensive work up that included blood cultures, chest X-rays and gallium scan. The transplanted kidney was thought to be the source of infection and she underwent transplant nephrectomy with complete resolution of her symptoms. She was switched to haemodialysis after the surgery. The patient remained off corticosteroids and immunosuppressive agents for more than a year.

While on haemodialysis, she had a recurrence of high-grade fever with night sweats, weight loss and nonspecific diarrhoea. Physical examination was unremarkable. All infectious work up, including chest X-rays, blood cul-

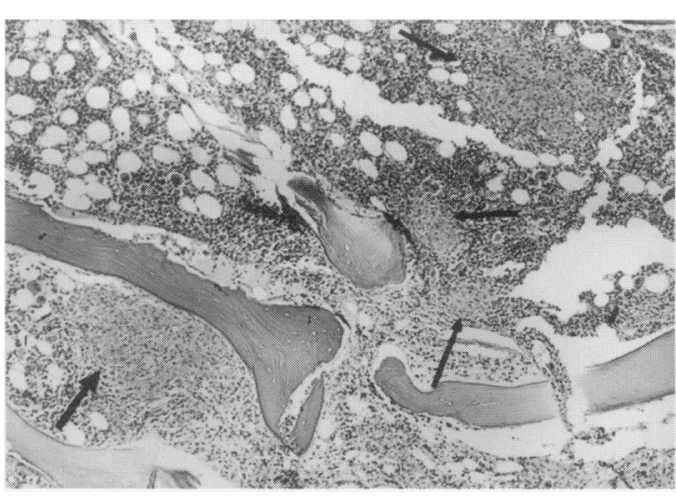

Figure 1 Histological appearance of bone marrow with noncaseating granuloma (short arrow) of Histoplasma capsulatum infection, bone marrow biopsy material from posterior iliac crest, before steroid therapy. Long arrow indicates granuloma (Hematoxylin, Eosin X 11)

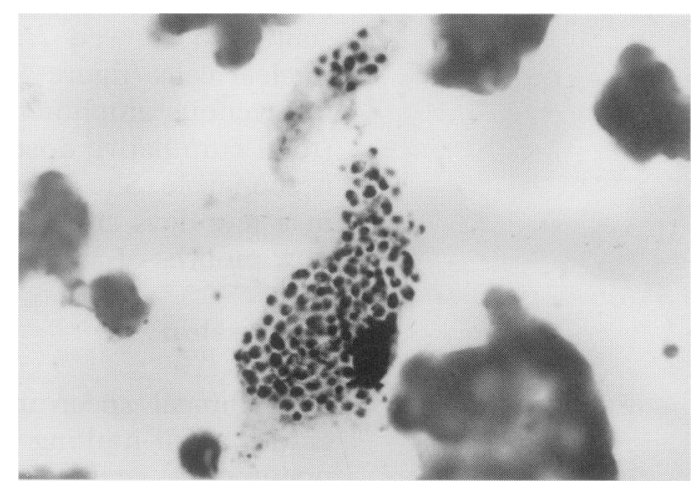

Figure 2 Intracytoplasmic appearance of Histoplasma capsulatum in a mononuclear cell within bone marrow aspirate smear preparation, after initiation of steroid therapy (Wright \& Giemsa, X 700) 
Figure 3 Histological appearance of bone marrow aspirate with rare microgranuloma $(-)$ of Histoplasma capsulatum infection, after initiation of steroid therapy (Hematoxylin, Eosin, X 230)
Figure 4 Intracytoplasmic appearance of Histoplasma capsulatum within a mononuclear cell in the peripheral blood smear, buffy coat preparation (Wright \& Giemsa, X 700)
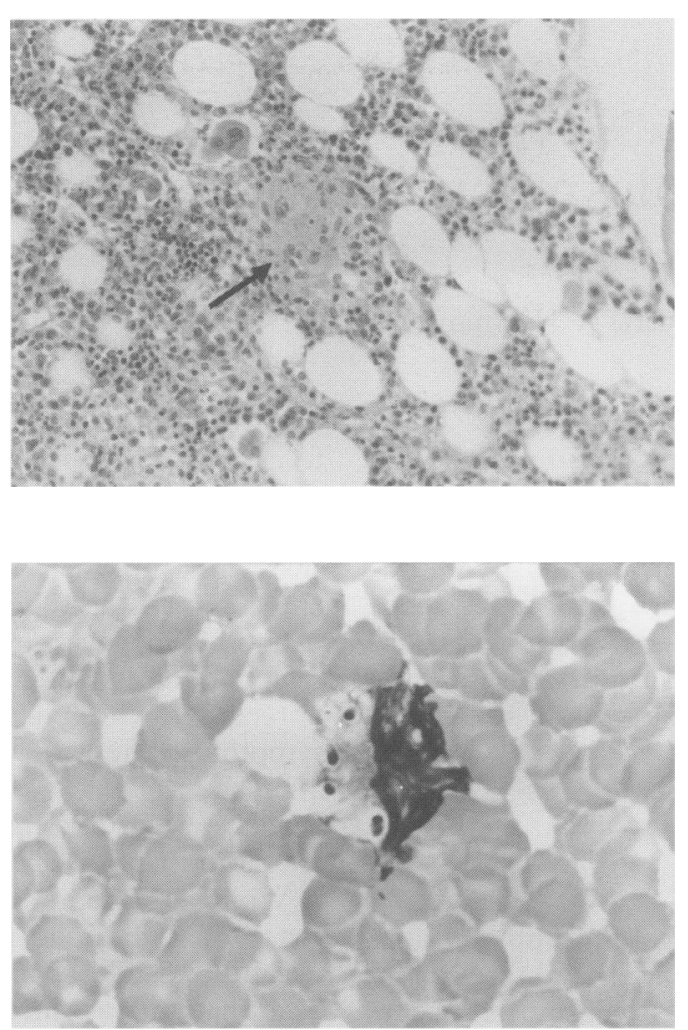

tures, stool studies, HIV and Mantoux test were negative. Gallium scan revealed no activity in the lung or any other organ. Bone marrow aspiration and biopsy revealed multiple noncaseating granulomas (figure 1). No organisms were seen. Mycobacterial and fungal cultures were done and she was started on high dose steroids for presumed recurrence of sarcoidosis.

She showed improvement, but within a week, fever and night sweats recurred, accompanied by hepatomegaly and abnormal liver function tests. Disseminated tuberculosis was suspected and, pending mycobacterial culture report, she was started on triple drug antituberculosis therapy. In the ensuing week her condition deteriorated; she developed leucopenia and thrombocytopenia with worsening liver function. Cultures remained negative. A repeat bone marrow aspirate and biopsy revealed numerous Histoplasma capsulatum within the reticuloendothelial cells (figure 2). There was rare granuloma (figure 3). A peripheral blood smear stained with Wright stain also showed Histoplasma within mononuclear cells (figure 4 ). The patient was given intravenous amphotericin B for three months (total cumulative dose $1.5 \mathrm{~g}$ ) followed by oral itraconazole. She responded dramatically within a few days and recovered completely after four months of antifungal therapy.

\section{Discussion}

The clinical spectrum of histoplasmosis includes a self-limiting influenza-like syndrome, pneumonia, mediastinal and pleural fibrosis, chronic lung cavities, histoplasmomas, miliary disease and disseminated, often fatal illness. ${ }^{1}$ Histoplasmosis may mimic sarcoidosis clinically or histologically but fungal superinfection is rare. ${ }^{4}$ The relationship of sarcoidosis and histoplasmosis was well described by Wheat et $a \tilde{P}$ in their review of 11 patients with sarcoidosis accompanied by laboratory evidence of histoplasmosis. They concluded that sarcoidosis and histoplasmosis are similar in many respects, clinically as well as radiographically. Noncaseating granulomas, the hallmark of sarcoidosis, are also seen in tissues from patients with histoplasmosis. ${ }^{6}$ Cases misdiagnosed as sarcoidosis have been described in which Histoplasma capsulatum was subsequently identified in tissues. $1,4,7,8$

Disseminated histoplasmosis is usually chronic or subacute. The subacute form occurs in apparently healthy individuals with mild parasitization. The histopathologic lesion is a well-formed noncaseating granuloma without any organism (figure 1). This kind of presentation can disguise as sarcoidosis. Sharma described an oriental man with granulomatous disease of the liver that mimicked sarcoidosis. ${ }^{1}$ The patient actually had histoplasmosis, which was finally diagnosed after eight weeks, with a culture of biopsied liver tissue.

Our patient is an excellent example of histoplasmosis masquerading as sarcoidosis. The bone marrow finding of noncaseating granuloma in the absence of any visible organisms in a patient with a previous history of sarcoidosis prompted a quick diagnosis of a recurrence 'of sarcoidosis. Since cultures of fungi and mycobacteria usually take weeks, the correct diagnosis was delayed. The continued deterioration in the clinical condition of the patient with development of progressive hepatosplenomegaly and pancytopenia while undergoing steroid therapy raised the spectre of a missed infection, which led to the repeat bone marrow biopsy. This provided the correct diagnosis and saved the patient's life. However, as it turned out, a careful review of Wrightstained peripheral blood smear or buffy coat (figure 4) would have yielded the diagnosis without any other invasive procedure. Although most of the immunological para-

\begin{tabular}{|l|}
\hline Pulmonary histoplasmosis: clinical \\
spectrum \\
\hline Acute \\
- asymptomatic \\
- self-limiting influenza-like syndrome \\
- severe dyspnea \\
Chronic \\
- granulomania \\
- mediastinal and pleural fibrosis \\
- chronic lung cavities \\
- 'Buckshotat' calcifications \\
Chest X-ray \\
- normal \\
- one or two patchy infiltrates \\
- diffuse miliary opacities \\
- pleural effusion and cavitation are uncommon \\
\hline
\end{tabular}




\begin{tabular}{|l|}
\hline Learning points \\
\hline - sarcoidosis may be misdiagnosed in patients \\
with histoplasmosis \\
- histoplasmosis may be a concomitant infection \\
in patients with sarcoidosis \\
- histoplasmosis may induce a chronic \\
inflammatory illness such as sarcoidosis \\
\hline
\end{tabular}

Box 2 meters were normal, the patient may have had underlying immunodeficiency since she was on haemodialysis.

In summary, the occurrence of histoplasmosis in patients with sarcoidosis requires careful evaluation. Active histoplasmosis must be carefully excluded in patients suspected to have sarcoidosis, as corticosteroid treatment may result in rapid progression of disseminated histoplasmosis in patients misdiagnosed as having sarcoidosis. ${ }^{9}$
1 Sharma OP. Histoplasmosis: a masquerader of sarcoidosis. Sarcoidosis 1991; 8: 10-13.

2 Goodwin RA, Shapiro IL, Thurman GH, et al. Disseminated histoplasmosis: clinical and pathological correlation. Medicine (Baltimore) 1980; 59: 1-33.

3 Tebib JG, Piens MA, Guillaux M, Colomb D, Garin JP, Tete R. Sarcoidosis possibly predisposing to disseminated Tete $R$. Sarcoidosis possibly predisposing
histoplasmosis. Thorax 1988; 43: 73-4

4 Winterbauer RH, Kraemer KG. The infectious complicaWinterbauer RH, Kraemer KG. The infectious complica-
tion of sarcoidosis. Arch Intern Med 1976; 136: 1156-62. 5 tion of sarcoidosis. Arch Intern Med 1976; 136: 1156-62. 5 Wheat JL, French MLV, Wass WL. Sarcoid like manifesta-
tion of histoplasmosis. Arch Intern Med 1989; 149: 2421 - 5 .
6 Sathapatayavongs B, Batteiger BE, Wheat LJ, Slama TG, Wass JL. Clinical and laboratory features of disseminated histoplasmosis during two large urban outbreaks. Medicine 1983; 62: $263-70$.

7 Israel HL, DeLamater E, Sones M, Willis WD, Mirmelstein

A. Chronic disseminated histoplasmosis. Am $\mathcal{f}$ Med 1952; 12: $252-60$.

8 Reimann HA, Price AH. Histoplasmosis resembling sarcoidosis. Trans Assoc Am Physicians 1949; 62: 112-5.

9 Sills M, Schwartz A, Weg JG. A consequence of progressive dissemination in the index case after steroid therapy. Ann Intern Med 1973; 79: 221-4.

\title{
Selenium-induced thyroid dysfunction
}

\author{
Lorenz C Hofbauer, Christine Spitzweg, Roland A Magerstädt, Armin E Heufelder
}

Division of

Endocrinology,

Medizinische Klinik,

Klinikum Innenstadt,

Ludwig-Maximilians-

Universität, Ziemssen-

straße 1, D-80336

Munich, Germany

LC Hofbauer

C Spitzweg

RA Magerstädt

AE Heufelder

Correspondence to Armin E. Heufelder, MD

Accepted 7 March 1996

\begin{abstract}
Summary
Administration of the anti-oxidative trace element selenium is currently being evaluated for its benefits in patients with inflammatory diseases. However, little is known about the risks of selenium. We report on a patient in whom, along with standard therapy, administration of large intravenous doses of selenite for sepsis secondary to pneumonia resulted in development of marked hypothyroidism. In addition, severe iodine deficiency was noted, and supplementation with iodine led to normalisation of thyroid function.
\end{abstract}

Keywords: selenium, iodine deficiency, hypothyroidism, euthyroid sick syndrome

The clinical benefit of selenium, an essential trace element with anti-oxidative effects, is currently under investigation..$^{1,2}$ Target compounds containing selenium include the antioxidative enzyme glutathione peroxidase and type I deionidase which catalyzes hepatic thyroxine $\left(T_{4}\right)$ to triiodothyronine $\left(T_{3}\right)$ conversion. ${ }^{3}$ We report on a patient who developed marked hypothyroidism following administration of large doses of selenium. Supplementation with iodide effectively restored normal thyroid function.

\section{Case report}

A 66-year-old man with chronic glomerulonephritis and no history of thyroid disorder was admitted for treatment of severe pneumonia following removal of a tongue cancer. Laboratory results showed an elevated white blood cell count of $15 \times 10^{9} / 1$ with a left shift, and elevated creatinine $(309.4 \mu \mathrm{mol} / \mathrm{l})$ and blood urea nitrogen $(29.6 \mathrm{mmol} / \mathrm{l})$ levels. Thyroid function tests revealed serum levels of thyroidstimulating hormone (thyrotropin) of $2.0 \mathrm{mIU} /$ 1 (normal $0.4-4.0$ ), free $T_{3}$ of $1.7 \mathrm{ng} / \mathrm{l}(2.3-$ 4.2 ), and free $T_{4}$ of $0.5 \mathrm{mg} / 1$ (normal $0.8-1.8$ ), consistent with euthyroid sick syndrome. Thyroid auto-antibodies were negative. Shortly

Side-effects of selenium
- odour of garlic on the breath
- fatigue, nausea
- diarrhoea, abdominal pain
- red discolouration at the base of the nails
- pulmocia
- polyneury oedema
- increased fT3/fT4 ratio, hypothyroidism (in the
presence of iodine deficiency)
- infertility

